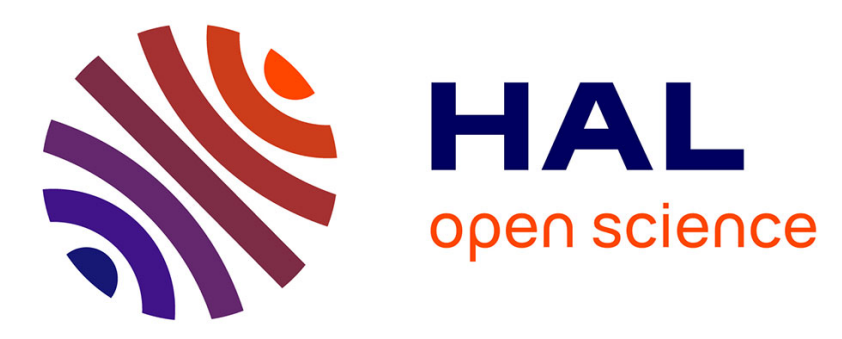

\title{
Enhancing Variable Friction Tactile Display using an ultrasonic travelling wave
}

Sofiane Ghenna, Eric Vezzoli, Christophe Giraud-Audine, Frédéric Giraud, Michel Amberg, Betty Lemaire-Semail

\section{- To cite this version:}

Sofiane Ghenna, Eric Vezzoli, Christophe Giraud-Audine, Frédéric Giraud, Michel Amberg, et al.. Enhancing Variable Friction Tactile Display using an ultrasonic travelling wave. IEEE Transactions on Haptics (ToH), 2016, 10 (2), pp.296 - 301. 10.1109/TOH.2016.2607200 hal-01425156

\section{HAL Id: hal-01425156 \\ https://inria.hal.science/hal-01425156}

Submitted on 3 Jan 2017

HAL is a multi-disciplinary open access archive for the deposit and dissemination of scientific research documents, whether they are published or not. The documents may come from teaching and research institutions in France or abroad, or from public or private research centers.
L'archive ouverte pluridisciplinaire HAL, est destinée au dépôt et à la diffusion de documents scientifiques de niveau recherche, publiés ou non, émanant des établissements d'enseignement et de recherche français ou étrangers, des laboratoires publics ou privés. 


\title{
Enhancing Variable Friction Tactile Display using an ultrasonic travelling wave
}

\author{
Sofiane Ghenna, Eric Vezzoli, Christophe Giraud-Audine , Member, IEEE, Frederic Giraud \\ , Member, IEEE, Michel Amberg, and Betty Lemaire-Semail, Member, IEEE
}

\begin{abstract}
In Variable Friction Tactile Displays, an ultrasonic standing wave can be used to reduce the friction coefficient between a user's finger sliding and a vibrating surface. However, by principle, the effect is limited by a saturation due to the contact mechanics, and very low friction levels require very high vibration amplitudes. Besides, to be effective, the user's finger has to move. We present a device which uses a travelling wave rather than a standing wave. We present a control that allows to realize such a travelling wave in a robust way, and thus can be implemented on various plane surfaces. We show experimentally that the force produced by the travelling wave has two superimposed contributions. The first one is equal to the friction reduction produced by a standing of the same vibration amplitude. The second produces a driving force in the opposite direction of the travelling wave. As a result, the modulation range of the tangential force on the finger can be extended to zero and even negative values. Moreover, the effect is dependant on the relative direction of exploration with regards to the travelling wave, which is perceivable and confirmed by a psycho-physical study.
\end{abstract}

Index Terms-Travelling wave, Friction Modulation, tactile stimulator and display.

\section{INTRODUCTION}

$\mathrm{T}$ HE control of the friction between a finger and a flat surface can be performed through different techniques, mainly electrovibration and ultrasonic vibration. The first relies on the application of a high voltage between the finger and an insulated electrode. The electrostatic attraction which is produced increases the normal force and, consequently, the friction force [1], [2]. The second uses a standing wave obtained at a resonance of a plate within the ultrasonic range. The finger sliding on it experiences a reduction of the friction coefficient if the amplitude of vibration is in the micrometer range. The effect has been firstly explained as a squeeze film effect [3], [4]; late evidence based on the fluidodynamic interaction explains the features of the friction reduction [5] and points toward a mechanical explanation [6] involving intermittent contact [7]. The induced sensations of the two techniques are similar [8] and the implementation of position tracking techniques allows to simulate simple texture rendering through the spatiotemporal modulation of the vibration [4].

So far, these techniques do not produce an active force on the finger. It means that they need a relative motion of the fingertip on the plate to increase or decrease the friction. To address this limitation [9] uses a surface haptic device which vibrates the touched surface simultaneously in both normal and lateral directions. The resulting elliptical surface motion produces the active force. The normal-bending and lateral-longitudinal resonances of a glass plate, and

- S. Ghenna and E.Vezzoli are with the L2EP-IRCICA Laboratory, University of Lille 1, Lille, 59650 France. E-mail: surname.name@ed.univ-lille.fr

- F. Giraud,M. Amberg and B.L. Semail, are with the L2EP-IRCICA Laboratory, University of Lille 1, Lille, 59650 France.

E-mail:surname.name@univ-lille.fr

- C. Giraud-Audine, is with Ecole nationale superieure d'arts et Métiers, Lille, 59000, France

E-mail: christophe.giraud-audine@ensam.eu its driving piezoelectric system, are arranged to occur at the same frequency. The relative phase of these vibration modes is used to control the magnitude and direction of the lateral force. Alternatively, a travelling wave can be used instead of a standing wave. In [11], a travelling Lamb wave tactile display has been proposed, to indicate smooth or braking sensation, using the stator of a travelling wave motor. The authors modelled the lateral stretching force as proportional to the relative velocity between finger and the surface without measuring the generating force.

In this paper, we propose a method to create a travelling lamb wave in a finite beam to enhance the tactile interaction with a variable friction display. In the first part, we describe how to obtain the travelling wave, and how it can produce an active force. In the second part, experimental study and comparison between the use of standing and travelling wave in tactile display is provided, exploring the influence of vibration amplitude, applied normal force and speed. Finally, a psychophysical validation is presented.

\section{THEORETICAL CONSIDERATIONS}

\subsection{Travelling wave generation}

In [12] two transducers were used to generate a travelling wave in a finite beam. One transducer is used to produce the travelling wave, while the other absorbs it to prevent the formation of standing wave. This method requires impedance matching between the beam and a transducer, as described in [13].

The second method [14] is based on the excitation of two successive flexure modes of the beam. Indeed, the frequency response of a beam features several modes such as represented on Fig.1 which highlights four modes of vibration.

By exciting the beam at an intermediate frequency between two modes (denoted $f_{\text {middle }}$ on the same figure) the 


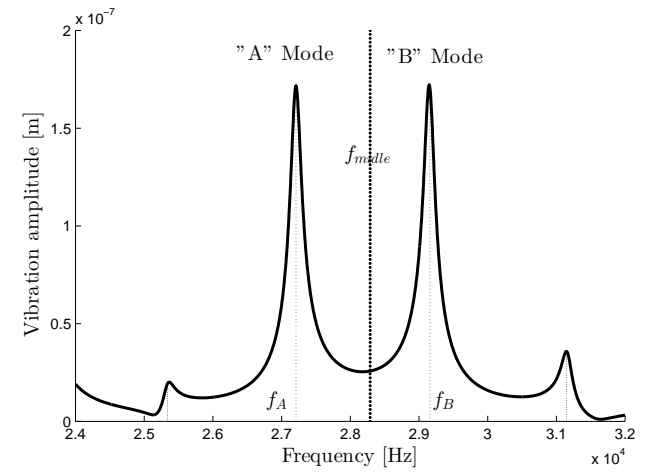

Fig. 1. Simulation of the frequency response of an aluminium beam of $600 \times 6 \times 6 \mathrm{~mm}^{3}$ for an excitation force amplitude of $0.5 \mathrm{~N}$ at $19 \mathrm{~mm}$ of the edge

modes $\mathrm{A}$ and $\mathrm{B}$ will be promoted. By imposing the same amplitude and a phase shift of $\pm 90^{\circ}$ on the two modes vibrations, a travelling wave is obtained [15]. In this work, we control the amplitude of each transducer in closed loop as explained in [16]; the advantages are to control the direction amplitude and direction of the travelling wave without any preliminary fitting procedures as in [15], and to mitigate external disturbances such as the pressure of a user's finger [17], [18]. Figure 2 depicts a typical implementation of a system generating an ultrasonic travelling wave. In this implementation, the two piezoelectric transducers excite each mode in the normal direction.

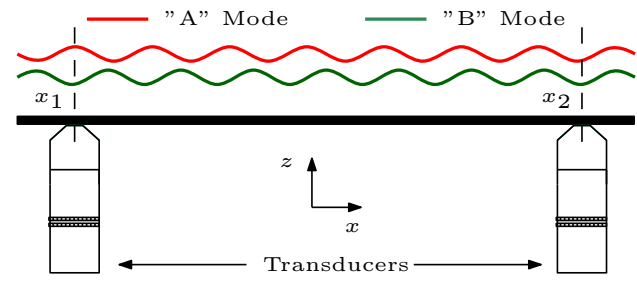

Fig. 2. A typical implementation of a system, and the two modes produced by the langevin transducers.

Therefore we proceed by giving the method to determine the vibration amplitude of the actuators. In steady state, the vibration amplitude on the beam $\underline{w}$, depends on both the normalized mode shapes $\phi_{n}(x)$, and their modal amplitude or modal participation factor $\underline{W}_{n}$ where $n$ is an integer, according to:

$$
\underline{w}(x, t)=\underline{W} e^{j \omega t}=\sum_{n=1}^{\infty} \frac{W_{n}}{\phi_{n}}(x) e^{j \omega t}
$$

$\underline{W}$ includes real and imaginary part of the vibration, at location $x, \omega$ is the angular frequency of vibration, and $j^{2}=-1$. Considering two dominant modes of vibration $A$ and $B$ and the positions of the actuators on the beam $x_{1}$ and $x_{2}$, the vibrations of the two actuators $W_{1}$ and $W_{2}$ are equal to the vibration of the beam at their position, which are given by:

$$
\begin{aligned}
& \frac{W_{1}}{W_{2}}=\underline{W}\left(x_{1}\right)=\underline{W}\left(x_{2}\right)=\underline{W_{A}} \phi_{A}\left(x_{1}\right)+\underline{W}_{A} \phi_{A}\left(x_{2}\right)+\underline{W}_{B} \phi_{B}\left(x_{1}\right) \\
& \left.\underline{W_{2}}\right)
\end{aligned}
$$

Introducing matrix notation, (2) becomes:

$$
\left(\begin{array}{l}
\underline{W}_{1} \\
\underline{W}_{2}
\end{array}\right)=\left(\begin{array}{ll}
\phi_{A}\left(x_{1}\right) & \phi_{B}\left(x_{1}\right) \\
\phi_{A}\left(x_{2}\right) & \phi_{B}\left(x_{2}\right)
\end{array}\right)\left(\begin{array}{l}
\underline{W}_{A} \\
\underline{W}_{B}
\end{array}\right)=\phi_{x_{1}, x_{2}}\left(\underline{W}_{B}\right)
$$

To obtain a travelling wave, the two modes $A$ and $B$ have to be superimposed, with the same modal amplitude, and phase shifted by $90^{\circ}$, as explained in figure 3 .

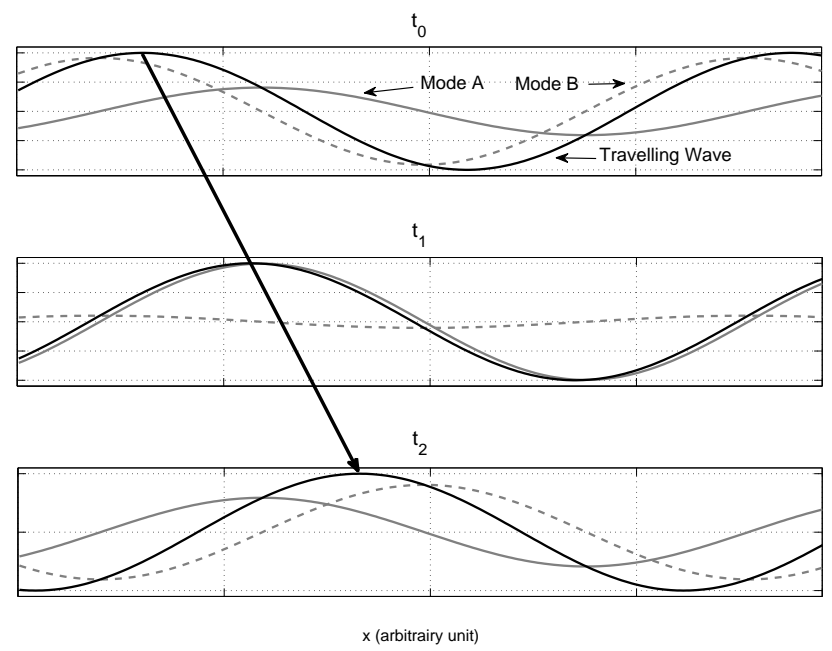

Fig. 3. The superposition of mode $A$ and mode $B$ at three different time stamps $t_{0}, t_{1}, t_{2}$, and the resulting travelling wave.

This leads to the following condition to obtain a travelling wave :

$$
\underline{W_{A}}= \pm j \underline{W_{B}}
$$

Hence, the reference signal for the transducers for an amplitude $W$ writes:

$$
\begin{aligned}
& W_{1}(t)=\operatorname{real}\left[W\left( \pm j \phi_{a}\left(x_{1}\right)+\phi_{b}\left(x_{1}\right)\right) e^{j \omega t}\right] \\
& W_{2}(t)=\operatorname{real}\left[W\left( \pm j \phi_{a}\left(x_{2}\right)+\phi_{b}\left(x_{2}\right)\right) e^{j \omega t}\right]
\end{aligned}
$$

The equations 5 shows that the references of each actuator do not necessarily have the same amplitude nor is their phase necessarily shifted by $90^{\circ}$ to obtain a travelling wave. The next section presents the modelling of the contact force produced by the travelling wave.

\subsection{Contact force modelling produced by the travelling wave}

The contact mechanism between the finger and the surface is modeled based on Fig. 5(a). When a pure travelling wave is generated along the beam, each point of the surface follows an elliptical trajectory as illustrated in Fig. 5(b). The horizontal component of the elliptical motion of particle leads to the generation of relative tangential speed between an object in contact and the beam as in [12], while the perpendicular component provides conditions for generating a squeeze film effect. The maximum particle speed at the top of the rail accordingly to [19] is given by:

$$
S_{h}=\frac{\pi W h \omega}{\lambda}
$$

where $W, h$ and $\lambda$ are respectively the vibration amplitude, thickness of the beam and wavelength. For the 


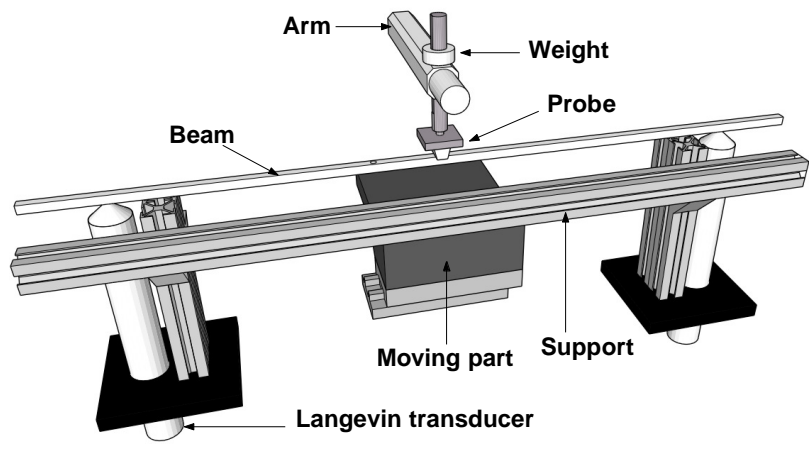

(a)

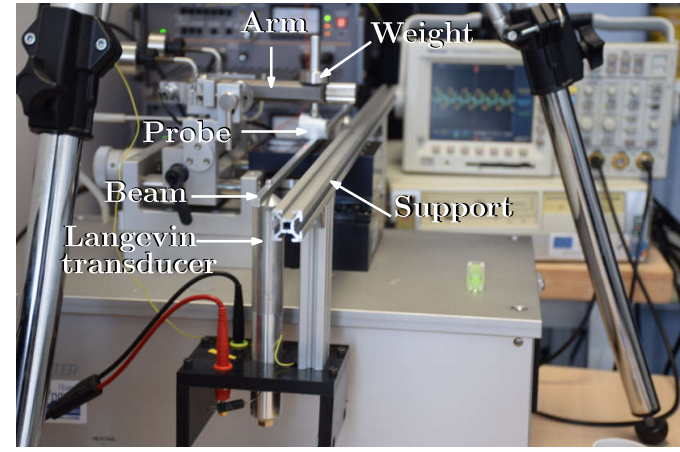

(b)

Fig. 4. Experiment set-up schematic, a beam is actuated by two Langevin transducers, fixed to the moving part of a Tribometer (a) and picture (b)

purpose of comparison, a vibration amplitude of $W=1 \mu \mathrm{m}$ gives a tangential speed of $80 \mathrm{~mm} / \mathrm{s}$. The study of the contact mechanisms shows that the total tangential force can be separated into two contribution [20]:

- a driving force denoted $F_{p}$ which propels the object in the direction opposite to the travelling wave,

- a friction force which depends on the velocity of the object, and which is opposite to the direction of its motion, and is denoted $F_{f}$.

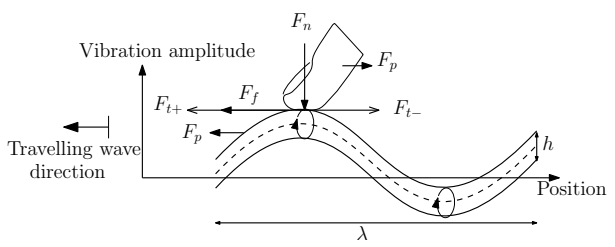

(a)

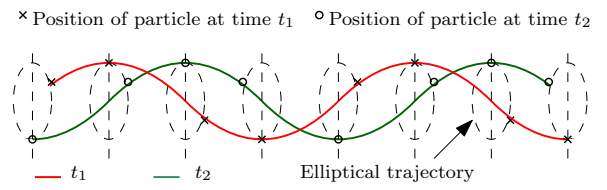

(b)

Fig. 5. (a) Principle of the force application by travelling wave. (b) Elliptical trajectory of the particles

These considerations allow us to write the resulting tangential force $F_{t}$ of the beam on a finger according to the relative direction of motion with the regards to the direction of the travelling wave. In this paper, the direction of the travelling wave remains the same, and we define $F_{t}$ positive when it acts against the movement of the finger. Therefore, $F_{p}$ and $F_{f}$ are always positive, and $F_{t}$ should write differently according to the direction of motion:

$$
F_{t+}=F_{f}-F_{p} \quad(7) \quad F_{t-}=F_{f}+F_{p}
$$

where $F_{t+}$ and $F_{t-}$ are tangential forces when the finger moves respectively in opposite direction and in direction of the travelling wave.

The contributions of each force is experimentally studied in the sequel and we present the experimental set-up in the next section.

\section{EXPERIMENTAL PROTOCOL}

\subsection{Experimental set-up}

To implement the generation of the travelling wave, a $600 \times$ $6 \times 6 \mathrm{~mm}^{3}$ aluminum beam was chosen. With those dimensions and material, the working frequency was $28.3 \mathrm{kHz}$, corresponding to the midpoint between the frequency of flexural mode $A$ and mode $B$, and the wavelength of the travelling wave was $\lambda=41 \mathrm{~mm}$. The experimental test bench is presented in Fig.4(a).

The beam is actuated by two Langevin transducers (FBL28252SSF-FC, Fujicera, Japan). The vibration amplitude of the two actuators is controlled through a DSP (TI 2812, Texas Instrument, USA) and two amplifiers (4051, HSA, Japan), the references for the control being previously calculated using Eq (5). The vibration amplitude of the beam was measured using an interferometer laser (OFV - 5000, Polytech, Germany). The whole system (beam with transducers) is attached to the support as shown in Fig.4(b). This support is fixed to the moving part of a Tribometer (TRB, CSI, Switzerland) allowing lateral friction force measurement. The parameters that can be selected are: the normal load and the linear sliding velocity. The apparatus is supported by a computer (data acquisition, monitoring, recording and analysis) software. The exploring length of the tribometer has been set to one wavelength due to its range limit of $60 \mathrm{~mm}$, with a sinusoidal exploration speed. In the paper, the friction is not measured with a fingertip, but with a probe which is validated in the next section.

\subsection{Probe Validation}

The probe interacting with the travelling wave has to behave as the human finger in the ultrasonic domain. To reflect the fact that the fingertip is a soft material, the probe which size is $16 \times 13 \times 10 \mathrm{~mm}^{3}$, is made of a silicone core with a grooved surface and rounded edges, covered with surgery tape to reproduce the mechanical behaviour of the fingertip [21].

In order to evaluate the used probe, we compared the friction modulation produced by a standing wave on it to the ones on a human finger [5] and a TangoPlus probe [22]. The results are presented in Fig.7 showing a similar friction modulation in the ultrasonic domain. 


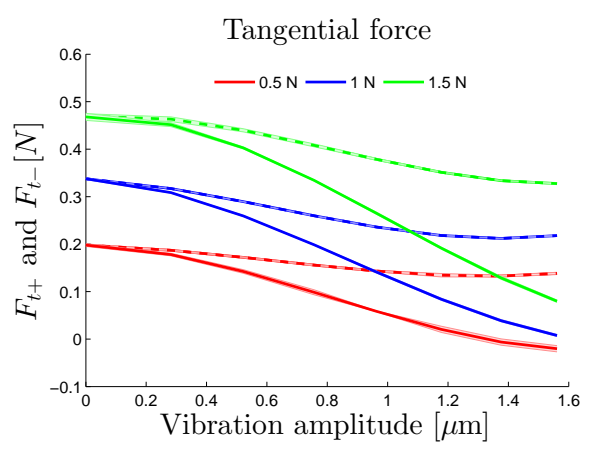

(a)

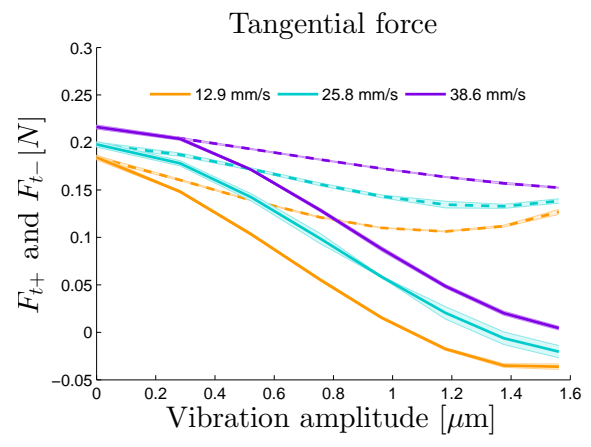

(d)

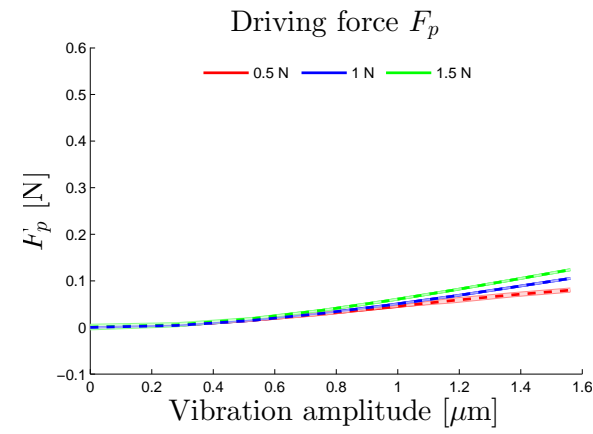

(b)

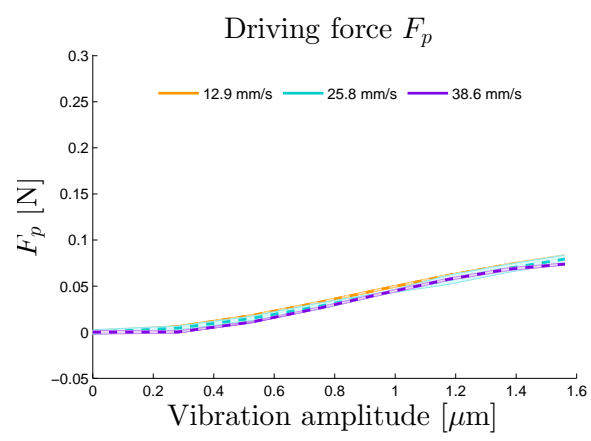

(e)

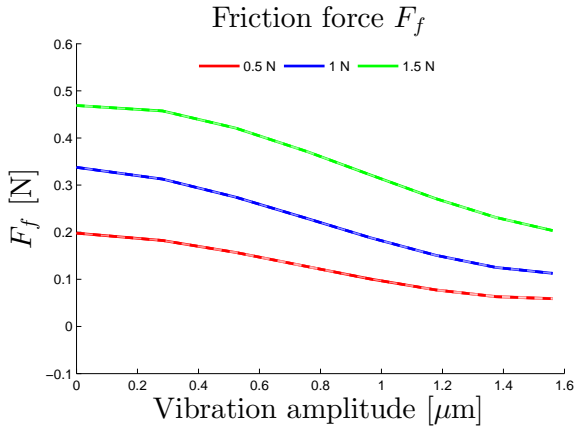

(c)

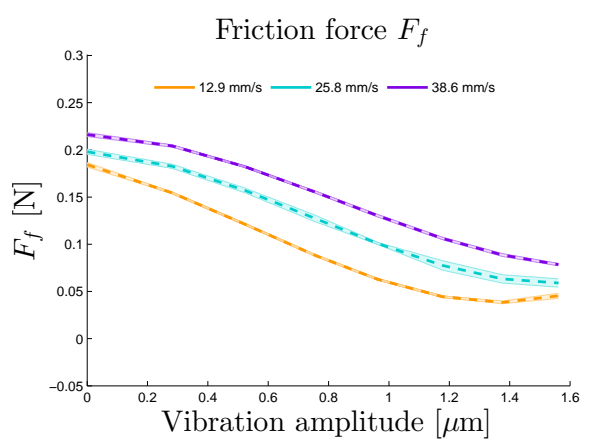

(f)

Fig. 6. Tangential forces (first column, $f_{t+}$ dashed line, and $f_{t-}$ plain line), driving forces (center column), friction forces (last column), for various normal force $F_{N}$ (top) and various velocities $v$ (bottom). The shadowed area near the measures represents the standard deviation for each measures.

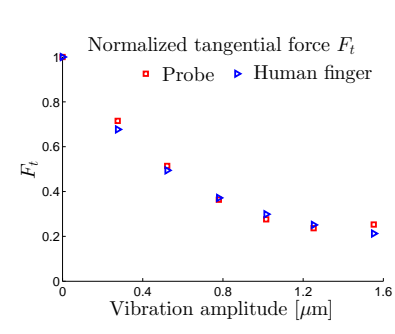

(a)

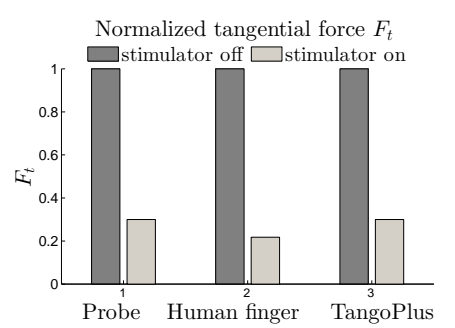

(b)
Fig. 7. (a) Comparison between the measured friction force for the used probe and the human finger in consistent condition of applied force and exploration speed [5]. (b) Comparison of the asymptotic friction reduction for the developed probe, human finger and the Tango plus probe [22]

Once the probe has been assessed, it has been used as a substitute to the human finger for the tribology measurements presented in the next section.

\subsection{Identification of $F_{p}$ and $F_{f}$}

The experimental set-up allows to measure the friction force for several speed $(v=12.9 \mathrm{~mm} / \mathrm{s}, v=25.8 \mathrm{~mm} / \mathrm{s}$ and $v=38.6 \mathrm{~mm} / \mathrm{s})$ and normal force $(F=0.5 \mathrm{~N}, F=1 \mathrm{~N}$ and $F=1.5 \mathrm{~N})$ conditions. We can also set different vibration amplitude from $W=0 \mu \mathrm{m}$ to $W=1.6 \mu \mathrm{m}$. For each operating condition, we averaged the measurements over 10 test runs.

Using (7) and (8) it is possible to extract the forces $F_{p}$ and $F_{f}$ from $F_{t+}$ and $F_{t-}$ by:

$$
F_{p}=\frac{F_{t-}-F_{t+}}{2} \quad \text { (9) } \quad F_{f}=\frac{F_{t-}+F_{t+}}{2}
$$

A tribological investigation has been done in order to evaluate the lateral friction forces, by changing experimental conditions: vibration amplitude, applied normal force and the exploration speed. The obtained results show that a direct influence on the lateral friction forces can be affected by these parameters.

\section{EXPERIMENTAL RESULTS}

In figure 6 we present the results for the different operating conditions; figures 6(a), 6(b) and 6(c) were obtained at a probe velocity of $25.8 \mathrm{~mm} / \mathrm{s}$ and normal forces of $F=0.5 \mathrm{~N}, F=1 \mathrm{~N}$ and $F=1.5 \mathrm{~N}$ while figures $6(\mathrm{~d})$, 6(e) and 6(f) were obtained at a normal force of $0.5 \mathrm{~N}$ and probe velocity of $v=12.9 \mathrm{~mm} / \mathrm{s}, v=25.8 \mathrm{~mm} / \mathrm{s}$ and $v=38.6 \mathrm{~mm} / \mathrm{s}$. Figures $6(\mathrm{a})$ and $6(\mathrm{~d})$ represent the evolution of the Lateral force $F_{t}$ measured by the tribometer (plain line: opposite direction to the travelling wave, dashed line: same direction as the travelling wave), 6(b) and 6(e) represent the actual driving force $F_{p}$ obtained using equation 9 and 6(c), 6(f) represent the calculated force $F_{f}$ using equation 10 .

\subsection{Influence of the vibration amplitude}

The figures 6(b), 6(c), 6(e), 6(f) show that the driving force $F_{p}$ increases with $W$, while $F_{f}$ decreases. The figures 6(a) 6(d) clearly show an influence of the progressive wave 
on the tangential force. The effect can be so high that the probe is propelled by the travelling wave. This happens for vibration amplitude $W$ above $1 \mu \mathrm{m}$, and for a displacement in opposite direction to the travelling wave.

By contrast, when the probe moves in the same direction as the travelling wave, the lateral force remains almost unchanged, because $F_{p}$ compensate the drop of the friction force $F_{f}$ produced by the vibrations. This highlights the fact that two antagonist effects exist in the contact mechanism: for one mechanism, vibrations reduce the friction as demonstrated by figure 6(c) and 6(f), while the other increases the driving force which can be added or subtracted by choosing direction of propagation. Although we could not obtain a higher apparent friction than the original one of the beam, we could cancel it. This is an improvement on a device based on standing waves only, which has a saturation on the friction reduction, as demonstrated in [6].

\subsection{Influence of the normal force}

As shown in [5], the normal force applied by the finger has a direct influence on the friction reduction for a device based on a standing wave. This also the case here, and the driving force $F_{p}$ and the friction force $F_{f}$ are both concerned, however, the main effect is on the friction force which is consistent with a coulombic force.

\subsection{Influence of the velocity}

The velocity of the probe has an influence on the tangential force $F_{T}$ in both directions. However, $F_{p}$ is almost unchanged, and thus, it can be concluded that the effect is mainly on the friction $F_{f}$ which again shows a coulombic force, but also presents a viscous component.

\subsection{Psychophysical study}

The perception of the consequences of the force applied by the travelling wave has been evaluated with a psychophysical experiment, performed using two-alternatives forcedchoice method described in [23]. The assumption under test is that this force induces different friction reduction according to the relative speed of the travelling wave and the finger. Six participants (5 males, 1 female; age range: $19-32$ years) were asked to wash their hands and properly dry them before the experiment. Also, they were asked to touch the beam where a standing wave was produced in order to train them to friction reduction. Afterwards, during the test, only travelling waves were used, and their directions were randomly set. The exploration was allowed for 10 seconds for each case with a vibration amplitude of $1.2 \mu \mathrm{m}$. The participants were asked to indicate "which of the two directions had less friction". An answer was positively scored if the direction with the lowest tangential force was detected. The experience was repeated 10 times with a randomization of the trials. The results are summed up on figure 8 . The participants were able to perceive the force applied by the travelling wave, with an average success rate of $81.66 \%$ and a standard deviation of $16 \%$. The hypothesis test against the random answer was refused with a $p<0.05$ leading to accept the conclusion that the direction of the applied force by a travelling wave was perceived. Indeed,

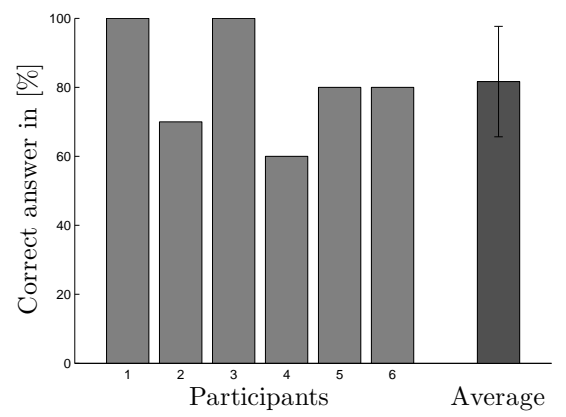

Fig. 8. Results of psychophysical testing

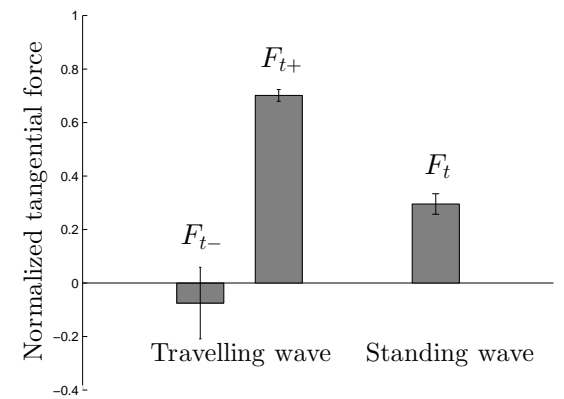

Fig. 9. Low friction level with a travelling wave and with a standing wave at $1.6 \mu \mathrm{m}$

the exercise is harder than telling the difference between "on" and "off", because the participants had to detect the change of friction which occurs with direction and also to detect the direction of propagation of the travelling wave. Hence, $100 \%$ is difficult to achieve. Considering this, we can conclude that when the finger explores the vibrating surface, it appears that one of the directions is more slippery in comparison with the opposite direction contrarily to the standing wave.

\section{Discussion}

The first feature of the travelling wave used in a variable friction tactile display is the higher contrast between low and high friction levels. In Fig. 9, the average of $F_{t}$ over the different experimental runs at maximum vibration amplitude are presented to highlight this point. For comparison purposes, the case of a standing wave is also presented on the same figure. The standing wave is obtained on the same experimental setup by setting $W_{B}=0$, and $W_{A}$ is controlled so as to always have the same amplitudes for standing and travelling wave. The control used in the experimental test bench exactly allows to compare the two cases together. The difference between the possible range between the extreme values of the net force on the probe approaches $70 \%$ of the force without vibration. It is also worth noticing that a negative net force can be produced by the travelling wave.

Moreover, the travelling wave offers the possibility to differentiate the feeling according to the direction of the exploration, this is shown on the same figure and confirmed by the psychophysical study.

So far the existence of the two contributions of $F_{p}$ and $F_{f}$ has been postulated. The behavior of the friction force $F_{f}$ 


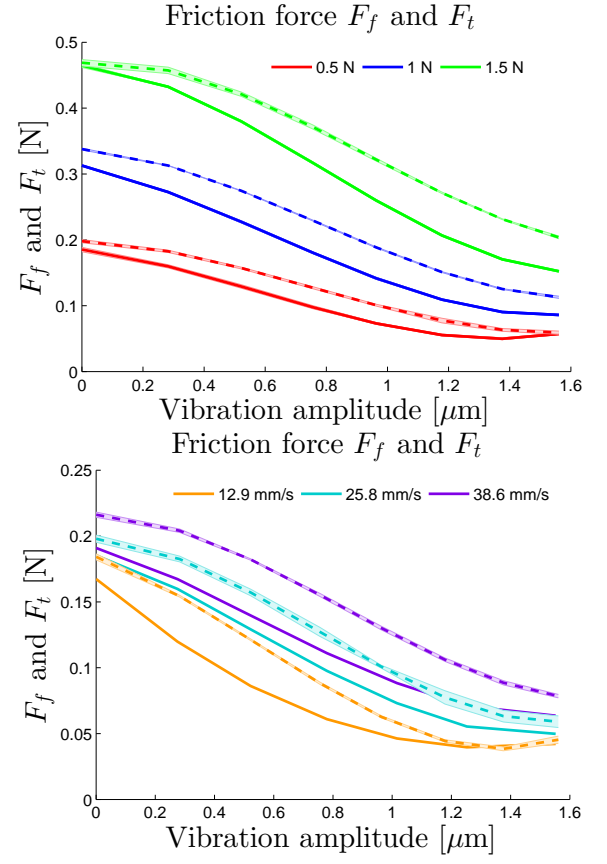

Fig. 10. Tangential forces for a standing wave (plain line) compared to the friction force $F_{f}$ of the travelling wave (dashed line) for several normal force (top) and velocities (bottom) conditions.

has been clearly linked to a coulombic friction with viscous components, due to its dependence on the normal force and the velocity of the exploration. To further investigate this point, the figures 10 superimpose the force $F_{f}$ obtained previously and compare it to tangential force obtained with a pure standing wave.

The figures clearly show that the estimated force $F_{f}$ on a travelling wave is equal to the tangential force produced by a standing for similar operating conditions. This demonstrates that the tangential force in the case of a travelling wave is the result of the superimposition of two contributions :

- The friction force which is equal to the effect of the standing wave which has been extensively studied,

- The driving force which is specific to the travelling wave and can be attributed to the elliptical motion at the surface of the beam.

This decomposition into two phenomena give some insight that could guide the design of tactile devices using travelling waves. Properties of the design such as material, geometry, wavelength, frequency could be exploited to promote the driving force $F_{p}$ over $F_{f}$. For example Eq (6) shows that it is necessary to increase beams thickness to promote the effect in the tangential axis. These points, however, require further investigation.

\section{Conclusion}

In this paper, the effect of an ultrasonic travelling wave on the friction force has been measured and the estimation of the applied force has been proposed for a finger pressure and an exploration speed compatible with touch exploration. The results confirmed the possibility to obtain an asymmetry of forces for a probe exploring the surface according to the direction of the travelling wave. The contrast of the friction is more important in the case of a travelling wave, compared to the standing wave. For extreme vibration amplitude, the tangential force can even reach a negative value and thus drag the finger. The influence of the velocity and the applied force has been reported.

Future work should focus on the design of new devices using a travelling exploiting two modes of vibration, with a larger exploring area, and the tracking of a user's finger to create spatio-temporal stimuli.

\section{ACKNOWLEDGMENTS}

This work has been carried out within the framework of the project StimTac of IRCICA (institut de recherche sur les composants logiciels et matériel pour la communication avancé), and the Project Mint of Inria. Authors thank FP7 Marie Curie Initial Training Network PROTOTOUCH, grant agreement No. 317100 for founding

\section{REFERENCES}

[1] K. A. Kaczmarek, K. Nammi, A. K. Agarwal, M. E. Tyler, S. J. Haase, et D. J. Beebe, "Polarity effect in electrovibration for tactile display," IEEE Trans. Biomed. Eng., vol. 53, no 10, p. 20472054 , oct. 2006.

[2] Bau, O., Poupyrev, I., Israr, A., Harrison, C.: TeslaTouch: electrovibration for touch surfaces. In: Proceedings of the 23rd Annual ACM Symposium on User Interface Software and Technology, pp. 283292, New York, 2010

[3] T. Watanabe and S. Fukui, A method for controlling tactile sensation of surface roughness using ultrasonic vibration, in IEEE International Conference on Robotics and Automation, vol. 1, pp. 11341139 vol.1. Nagoya, 1995.

[4] M. Biet, G. Casiez, F. Giraud, and B. Lemaire-Semail, Discrimination of Virtual Square Gratings by Dynamic Touch on Friction Based Tactile Displays, in symposium on Haptic interfaces for virtual environment and teleoperator systems, pp. 4148, Nevada, USA,2008.

[5] T. Sednaoui, E. Vezzoli, B. Dzidek, B. Lemaire-Semail, C. Chappaz, and M. Adams, Experimental evaluation of friction reduction in ultrasonic devices, in IEEE World Haptics Conference (WHC), pp. 3742, Chicago, USA, 2015

[6] E. Vezzoli, B. Dzidek, T. Sednaoui, F. Giraud, M. Adams, and B. Lemaire-Semail, Role of fingerprint mechanics and non-Coulombic friction in ultrasonic devices, in 2015 IEEE World Haptics Conference (WHC), pp. 4348, Chicago, USA, 2015

[7] M. Wiertlewski, R. F. Friesen, and J. E. Colgate, Partial squeeze film levitation modulates fingertip friction, PNAS, vol. 113, no. 33, pp. 92109215, 2016.

[8] E. Vezzoli, W. Ben Messaoud, M. Amberg, F. Giraud, B. LemaireSemail, and M.-A. Bueno, Physical and Perceptual Independence of Ultrasonic Vibration and Electrovibration for Friction Modulation, IEEE Transactions on Haptics, vol. 8, no. 2, pp. 235239 , Apr. 2015.

[9] X. Dai, J. E. Colgate, and M. A. Peshkin, LateralPaD: A surfacehaptic device that produces lateral forces on a bare finger, in IEEE Haptics Symposium (HAPTICS), pp. 714, Vancouver, 2012

[10] S.-Y. Kim, J.-O. Kim, and K. Kim, Traveling vibrotactile wave - a new vibrotactile rendering method for mobile devices, IEEE Transactions on Consumer Electronics, vol. 55, no. 3, pp. 10321038, Aug. 2009.

[11] M. Biet, F. Giraud, F. Martinot, and B. Semail, "A Piezoelectric Tactile Display Using Travelling Lamb Wave," Proc. Eurohaptics Conf, pp. 567-570, Paris 2006.

[12] T. Sashida and T. Kenjo, An introduction to ultrasonic motors. Oxford: New York: Clarendon Press; Oxford University Press, 1993.

[13] M. Kurosawa and S. Ueha, High speed ultrasonic linear motor with high transmission efficiency, Ultrasonics, vol. 27, no. 1, pp. 3944, Jan. 1989.

[14] B.-G. Loh and P. . Ro, "An object transport system using flexural ultrasonic progressive waves generated by two-mode excitation," IEEE Transactions on Ultrasonics, Ferroelectrics, and Frequency Control, vol. 47, no. 4, pp. 994-999, Jul. 2000. 
[15] A. Minikes, R. Gabay, I. Bucher, and M. Feldman, "On the sensing and tuning of progressive structural vibration waves," IEEE Transactions on Ultrasonics, Ferroelectrics, and Frequency Control, vol. 52, no. 9, pp. 1565-1576, Sep. 2005.

[16] S. Ghenna, F. Giraud, C. Giraud-Audine, M. Amberg, and B. Semail, Modelling, identification and control of a Langevin transducer, in 2015 IEEE International Workshop of Electronics, Control, Measurement, Signals and their Application to Mechatronics (ECMSM), pp. 16. Leberec, 2015.

[17] F. Giraud, C. Giraud-Audine, M. Amberg, and B. Lemaire-Semail, "Vector control method applied to a traveling wave in a finite beam," IEEE Transactions on Ultrasonics, Ferroelectrics, and Frequency Control, vol. 61, no. 1, pp. 147158, Jan. 2014.

[18] S. Ghenna, F. Giraud, C. Giraud-Audine, M. Amberg, B. Semail, Modelling and control of a travelling wave in a finite beam, using multi-modal approach and vector control method, in IEEE International Frequency Control Symposium, European Frequency and Time Forum,(IFCS-EFTF), Danvers, USA, 2015.

[19] J. Maas, P. Ide, N. Frohleke, and H. Grotstollen, Simulation model for ultrasonic motors powered by resonant converters, in , Conference Record of the IEEE Industry Applications Conference. Thirtieth IAS Annual Meeting, IAS 95, 1995, vol. 1, pp. 111120 vol.1

[20] J. Wallaschek, Contact mechanics of piezoelectric ultrasonic motors, Smart Mater. Struct., vol. 7, no. 3, p. 369, 1998.

[21] M.-A. Bueno, B. Lemaire-Semail, M. Amberg, and F. Giraud, Pile Surface Tactile Simulation: Role of the Slider Shape, Texture Close to Fingerprints, and the Joint Stiffness, Tribol Lett, vol. 59, no. 1, pp. 112, Jun. 2015.

[22] R. F. Friesen, M. Wiertlewski, M. A. Peshkin, and J. E. Colgate, Bioinspired artificial fingertips that exhibit friction reduction when subjected to transverse ultrasonic vibrations, in IEEE World Haptics Conference (WHC), pp. 208213, Chicago, USA, 2015

[23] L. A. Jones and H. Z. Tan, Application of Psychophysical Techniques to Haptic Research, IEEE Transactions on Haptics, vol. 6, no. 3, pp. 268-284, Jul. 2013. 